Service social

\title{
L'utilisation du plan de services individualisé en protection de la jeunesse
}

\section{Daniel Turcotte}

Volume 41, numéro 3, 1992

Intervenir en contexte d'autorité

URI : https://id.erudit.org/iderudit/706583ar

DOI : https://doi.org/10.7202/706583ar

Aller au sommaire du numéro

\section{Éditeur(s)}

École de service social de l'Université Laval

ISSN

1708-1734 (numérique)

Découvrir la revue

Citer cet article

Turcotte, D. (1992). L'utilisation du plan de services individualisé en protection de la jeunesse. Service social, 41(3), 25-39. https://doi.org/10.7202/706583ar
Résumé de l'article

Le développement de la pratique en matière d'application des mesures de protection passe par le recours à des stratégies d'action concertées qui mettent à contribution les intervenantes et les intervenants du réseau et des organismes communautaires. Une telle orientation s'appuie sur le recours à une démarche où ces acteurs unissent leurs efforts autour d'objectifs et de moyens définis collectivement dans le cadre d'un plan de services individualisé. Initialement utilisé en réadaptation, le plan de services individualisé tend à se généraliser à plusieurs domaines de pratique, dont celui de la protection de la jeunesse.

En raison des particularités que présente l'intervention en contexte d'autorité, son actualisation risque toutefois de se buter à certaines difficultés dans le domaine de la protection. Ce texte présente les réflexions de l'auteur sur ce sujet. 


\title{
L'utilisation du plan de services individualisé en protection de la jeunesse
}

\author{
Daniel TURCOTTE \\ Professeur, École de service social \\ Université Laval
}

L'entrée en vigueur de la Loi sur la protection de la jeunesse a profondément transformé les services sociaux à l'enfance et à la famille. Même si cette loi s'adresse à une clientèle d'exception, au fil des ans cette clientèle est devenue de plus en plus nombreuse et la nature des situations signalées plus problématique. Pour certains, comme Besharov (1986), I'augmentation du nombre de signalements, dont plusieurs se révèlent par la suite non fondés, constitue un problème important de l'application des mesures de protection de la jeunesse. D'autres, comme Rothery (1990), soulignent plutôt l'obstacle que représente la désillusion des intervenants devant le peu de succès de leurs interventions auprès des familles où un enfant est victime de mauvais traitements ou de négligence. Plusieurs estiment qu'ils n'ont pas le pouvoir de changer la réalité de ces familles, "puisque souvent les causes de leurs problèmes ont comme origine l'organisation de notre société » (Gagné, 1991 : 12).

Comme les familles maltraitantes font généralement face à des sources de stress multiples tout en ayant accès à des ressources de soutien déficientes (Garbarino et Stocking, 1980), elles sont particulièrement difficiles à aider. Les approches traditionnelles d'intervention ont généralement des effets limités auprès de ces familles et les résultats qu'obtiennent les intervenants sont 
rarement proportionnels aux énergies qu'ils investissent. Il y a là un terrain propice à l'épuisement professionnel et au désabusement.

Pour sortir de cette impasse, il est nécessaire de repenser les modes $d^{\prime}$ 'intervention auprès des familles en mettant en place des services fondés sur un partenariat entre les ressources formelles et informelles (Whittaker, 1986) et qui combinent l'aide concrète, la consultation psychosociale et la défense du client (Jones, Magura et Shyne, 1981). Selon Rothery (1990), quatre principes doivent servir de guide à la définition de l'intervention en matière de protection de l'enfance : 1) I'utilisation d'un ensemble de services coordonnés plutôt que le recours à une forme spécialisée d'aide, 2) l'accessibilité à un éventail de formes de soutien (soutien émotionnel, services concrets, aides éducatives variées, réseau d'entraide), 3) la priorité donnée aux compétences et aux forces de la famille plutôt que la résolution de ses conflits émotionnels et 4) la coordination des services. Une approche efficace du problème de la maltraitance ne peut donc reposer sur un seul intervenant; elle doit plutôt mettre à profit le plus large éventail possible de compétences et de ressources : « no one worker can provide a sufficient response to the needs of maltreating families » (Rothery, $1990: 7$ ).

La mise à contribution de plusieurs ressources pose toutefois le problème de la coordination des actions. L'option qui est privilégiée pour assurer cette coordination est le plan de services individualisé (PSI). Initialement mis au point dans le domaine de la réadaptation et promu au Québec par I'Office des personnes handicapées (OPHQ) au début des années 80, le PSI est actuellement considéré comme une mesure susceptible d'améliorer la pratique en protection de la jeunesse (Cohn et De Graaf, 1982 ; Harvey, 1988 ; Groupe de travail pour les jeunes, 1991 ; Groupe de travail sur I'application des mesures de protection de la jeunesse, 1991a ; Rothery et Cameron, 1990). Son actualisation dans ce domaine ne peut toutefois se faire sans tenir compte des conditions particulières de la pratique en contexte d'autorité. C'est là l'objet de ce texte qui porte sur les particularités de l'application du plan de services individualisé dans le domaine de l'intervention en protection de la jeunesse. La première partie présente un bref survol des principales définitions de la notion de plan de services individualisé et décrit les principes et les étapes qui doivent en guider l'élaboration. Par la suite, quelques conséquences de I'utilisation du PSI sur la pratique du travail social en contexte d'autorité sont soulevées. Enfin, en dernière partie, quelques pistes d'action visant à en faciliter l'intégration à la pratique sont suggérées.

\section{LE PLAN DE SERVICES INDIVIDUALISÉ}

Le concept de plan de services individualisé est une notion à multiples facettes, dont on trouve diverses définitions. Certains auteurs le présentent en 
mettant I'accent sur son objectif. Ils parlent alors d'un instrument couvrant I'ensemble des services qui doivent être rendus pour corriger une situation (Harvey, 1988a), d'un outil de planification et de coordination des services (CRSSSMM, 1989 ; Côté et al., 1989 ; Despins, 1990), d’un mécanisme qui relie et coordonne les parties d'un système de distribution de services (Austin, 1981 ; 1983), d'un moyen adapté aux personnes avec des besoins multiples (Pyke et al., 1991) ou, encore, d'un instrument intégrateur qui permet la planification et la concertation des services (ACSSQ, 1988).

D'autres auteurs insistent davantage sur sa démarche. Leur définition situe le PSI comme un processus de coordination des ressources (Lister, 1984), comme une méthode de planification et de coordination des services (Laurendeau, 1989, Labrecque-Marceau, 1987), comme une méthode pour assurer des services coordonnés, efficaces et efficients (Kemp, 1981 ; Intagliata et Baker, 1983) ou comme une démarche concertée de planification et de prestation des services (Groupe de travail sur l'application des mesures de protection de la jeunesse, 1991b).

D'autres, enfin, font surtout ressortir la philosophie qui se profile derrière le PSI et la perspective dans laquelle celui-ci doit s'inscrire. Leur définition le présente comme une approche de distribution des services (Cabot et al., 1987) qui suppose la continuité des services, la planification sur le plan de l'élaboration et de l'exécution d'un plan d'action et la coordination entre les différents acteurs (Roberts-DeGennaro, 1987). Selon cette optique, la notion de plan de services individualisé s'apparente au concept de « case management » que Rubin (1987 : 212) définit comme une approche de distribution de services qui vise à faire en sorte que les clients aux prises avec des problèmes multiples et complexes reçoivent tous les services dont ils ont besoin, de façon appropriée et au moment opportun.

Ces trois façons de définir le PSI ne sont pas contradictoires mais complémentaires, puisqu'elles en font ressortir les composantes essentielles, à savoir : son objectif, sa démarche et sa philosophie. On retrouve d'ailleurs ces composantes dans la définition proposée par le Groupe de travail sur l'application des mesures de protection de la jeunesse (1991b : 18).

Le plan de services individualisé est une démarche concertée qui, par la coordination des interventions et la participation active de la personne ou de son représentant, permet la planification et la prestation des services nécessaires pour répondre aux besoins de cette personne, afin de favoriser ou de maintenir son intégration tout en assurant sa protection.

Au point de vue opérationnel, le plan de services individualisé s'actualise dans un plan d'action qui, à partir d'une évaluation globale des besoins et des ressources de l'enfant et de sa famille, précise les buts, les objectifs particuliers, les moyens à mettre en œuvre et la durée prévisible de 
I'intervention. Il doit également faire mention de la contribution attendue de chacun des acteurs et préciser les mécanismes de révision et d'évaluation, de même que les modalités de coordination. Le PSI est donc plus qu'un plan d'action : c'est une stratégie de planification et de mise en application de mesures qui vise à offrir de meilleures conditions de vie à des clientèles à risque (Labrecque-Marceau, 1987).

\section{LES PRINCIPES ET LES ÉTAPES DU PSI}

Le plan de services individualisé s'inscrit dans une philosophie d'intervention qui met au premier plan la nécessité d'assurer l'accessibilité, la continuité et la complémentarité de services en vue d'apporter une réponse appropriée aux besoins multiples de la personne. Il se présente comme une mesure qui, en plus d'assurer une plus grande continuité des services, contribue à ce que les services rendus répondent à l'ensemble des besoins de la personne par une prestation adéquate, opportune et sans duplication de ces services (Rubin, 1987). Pour ce faire, certains principes doivent guider le contenu et la démarche d'élaboration du PSI. Ces principes sont:

1. l'évaluation de la personne dans une perspective globale qui tient compte non seulement de ses besoins, mais également de ses compétences et de ses ressources ;

2. l'élaboration d'objectifs axés sur la participation et l'intégration sociale ;

3. la mise à contribution des ressources familiales et des réseaux primaires et de la communauté tant au chapitre de la planification que de la prestation des services ;

4. le souci de favoriser la continuité, la complémentarité et l'efficacité des services ;

5. la participation active du client à l'élaboration du PSI ;

6. I'appropriation de la démarche par le client ;

7. I'engagement des collaborateurs éventuels par rapport à la nécessité d'actions concertées (ACSSQ, 1988).

\section{LA COORDINATION DES SERVICES}

Comme le plan de services individualisé met en scène un éventail de services souvent fournis par des organismes différents, la coordination est une activité 
centrale de sa mise en application. Considéré comme le « pivot du plan de services»(CSSQ, 1989 : 5.2), le coordonnateur a pour fonction principale de « mettre le client en relation avec le réseau des services et [ $\left.d^{\prime}\right]$ assurer la coordination de ceux dont il a besoin » (Labrecque-Marceau, 1987 : 112). La coordination est une fonction complexe qui comporte plusieurs volets. On trouve dans la littérature sur le sujet diverses façons de l'envisager.

Selon Moxley (1989), la coordination doit s'articuler autour de trois objectifs principaux : 1) augmenter la capacité du client à obtenir et à utiliser les services et les sources de soutien qui lui sont accessibles, 2) accroître la contribution des réseaux primaires et des organismes formels et 3) promouvoir l'efficacité de l'intervention en facilitant l'accès à des services de qualité. Selon Schilling et al. (1988), les trois activités principales du coordonnateur sont la représentation du client (advocacy), le courtage de services (brokering) et le développement de services naturels et formels en mesure de répondre aux besoins du client (networking). Pour d'autres auteurs, les fonctions principales du coordonnateur correspondent à l'évaluation, la planification, l'intervention, le courtage, la supervision et l'évaluation (Moxley, 1989 ; Rubin, 1987).

Au-delà des nuances, il se dégage que le coordonnateur est le représentant du client auprès des ressources de la communauté. En tant qu'intermédiaire entre le client et les ressources, la position du coordonnateur n'est cependant pas toujours confortable et la réalisation de son mandat de superviseur des services le place dans une position particulière par rapport aux autres intervenants. Comme le souligne Despins (1990) :

La coordination n'est pas une fonction simple ni facile à assumer. Elle exige à la fois de la diplomatie, de l'esprit d'initiative et de la fermeté dans l'application des décisions (p. 68).

Bien que peu de recherches aient été réalisées jusqu'à maintenant sur I'impact des plans de services individualisés, particulièrement dans le domaine de la protection de la jeunesse (Cohn et De Graaf, 1982 ; Franklin et al., 1987), en théorie on leur reconnaît plusieurs avantages tant pour les praticiens que pour les enfants.

Ils créent un contexte d'échanges qui sort les intervenants de leur isolement et enrichit le répertoire des ressources disponibles pour venir en aide à l'enfant. Ils favorisent une approche plus cohérente, ce dont les enfants, particulièrement ceux victimes d'abus, ont grandement besoin. La mise en présence des personnes significatives dans la prise en charge du dossier permet aussi un dévoilement des préoccupations et des inconforts de chaque intervenant (Groupe de travail pour les jeunes, $1991: 169$ ).

Ils comportent toutefois certains inconvénients. La lourdeur des tâches liées à la planification, à la sollicitation et à la coordination des services peut 
devenir un facteur d'épuisement pour les intervenants. En outre, les exigences de la coordination peuvent être sources d'insatisfaction et de frustration, particulièrement si les intervenants sont insuffisamment préparés pour assumer cette fonction. Il semble donc y avoir certaines conditions préalables à une utilisation efficace des plans de services.

La première est la nécessité pour l'intervenant d'avoir une connaissance étendue des ressources formelles et informelles de la communauté et de reconnaître l'apport de ce réseau dans la solution des problèmes que rencontrent les personnes en difficulté. Si cette condition est absente, la mise à contribution de ces ressources est illusoire et le PSI, sans intérêt. Une seconde condition tient à la nécessité d'adopter, au moment de l'évaluation, une perspective globale qui tient compte à la fois des forces et des besoins de la personne et qui met l'accent sur ses possibilités et ses compétences plutôt que sur ses limites. Le PSI s'inscrit dans un mode de pratique qui reconnaît l'importance d'intégrer les dimensions bio-psycho-sociales de l'intervention (Nadeau, 1989). Une troisième condition se rapporte à la nécessité de partager, avec la personne et avec les collaborateurs potentiels, les données de l'évaluation et de faire participer tous ces acteurs à la spécification des objectifs généraux, à la sélection des moyens d'action et à la détermination des ressources à utiliser. Cette participation apparait essentielle pour que chaque collaborateur en arrive, par la suite, à formuler un plan d'intervention qui soit compatible avec le plan de services. Établi en fonction du mandat et des compétences propres à chacun, ce plan d'intervention précise les moyens qui seront utilisés et situe leur mise en application dans le temps. Si le PSI fait appel à des décisions collectives pour la définition des besoins prioritaires, la spécification des objectifs et le choix des moyens d'action, la formulation et l'application des plans d'intervention résultent pour leur part de décisions individuelles :

La responsabilité d'orienter et de planifier les services requis est collective (...) [mais] chacun des établissements et organismes est appelé à dispenser les services et doit articuler et orienter son action en fonction du plan général (CRSSSMM, 1989 : 34).

Comme il s'agit d'une fonction essentielle dans l'actualisation du plan de services, certaines conditions se posent également en ce qui regarde la coordination. Ainsi, dans la mesure où la rigidité administrative constitue un des principaux obstacles auxquels doit faire face le coordonnateur (Johnson et Rubin, 1983), l'autorité que celui-ci peut exercer sur les ressources mises à contribution est une composante importante de l'efficacité du plan de services individualisé (Rubin, 1987). Cette autorité est essentielle pour contourner les obstacles qui réduisent l'accès aux services disponibles et pour être en mesure d'exercer une supervision convenable des services fournis.

Pourtant, l'autorité n'aura qu'une portée limitée si celui qui assure la coordination trouve peu de pertinence et d'intérêt à cette fonction. Or, même 
si les travailleurs sociaux sont généralement considérés comme les professionnels les mieux outillés pour assurer la coordination des plans de services (Segal et Baumohl, 1981), paradoxalement ils apparaissent plus ou moins intéressés par cette fonction qui correspond pourtant aux premières formes de travail social (Schilling et al., 1988). Les travailleurs sociaux sont réfractaires à l'idée d'assumer les tâches liées à la coordination.

Despite the strong conceptual similarities between social work and case management, the strongest deterrent to the prominence of social work in this area may be, paradoxically, the preferences of many social workers themselves. A large number of social workers in settings in which case management is appropriate appear to be indifferent to such work (Johnson et Rubin, 1983 : 52).

Même si l'intervention auprès de la personne dans son environnement constitue le principe de base du travail social, il semble difficile pour les intervenants de concilier l'intervention psychothérapeutique et l'aide concrète. Dans la réalité quotidienne, le discours du travail social sur l'importance d'avoir une perspective globale, sur la nécessité de fournir des services directs et sur l'exigence de défendre les intérêts du client cède le pas à la psychothérapie individuelle. Plusieurs travailleurs sociaux évaluent la coordination des services comme une pratique plus superficielle et moins professionnelle que la psychothérapie (Johnson et Rubin, 1983).

\section{LES CARACTÉRISTIQUES DE L'INTERVENTION EN CONTEXTE D'AUTORITÉ}

Ces considérations générales sur les conditions d'utilisation des plans de services individualisés sont susceptibles d'apparaître sous une teinte encore plus accentuée lorsque le domaine d'application est la pratique en contexte d'autorité telle qu'elle se présente actuellement dans le cadre de la Loi sur la protection de la jeunesse. Ce champ de pratique comporte des caractéristiques liées, notamment, à la spécificité de l'intervention de protection, à la segmentation des activités d'intervention, aux particularités organisationnelles et aux impératifs d'ordre légal qui imposent des contraintes particulières à l'actualisation du plan de services individualisé.

\section{La spécificité de l'intervention de protection}

Les principes à la base du plan de services individualisé soulignent, d'une part, la nécessité d'évaluer la situation de la personne en difficulté dans une perspective globale et compréhensive qui tient compte tout autant de ses compétences et de ses ressources que de ses limites et de ses besoins et 
affirment, d'autre part, l'importance de poursuivre des objectifs de développement personnel et d'intégration sociale. Dans le cadre de la Loi sur la protection de la jeunesse, le domaine de l'intervention se limite à l'espace restreint de la compromission de la sécurité ou du développement. Le champ d'investigation exploré au moment de l'évaluation est délimité par le jugement à poser en matière de compromission et par l'argumentation éventuelle de ce jugement au tribunal. Ainsi l'évaluation est souvent strictement centrée sur la réponse fournie aux besoins essentiels de l'enfant et sur la reconnaissance des aspects négatifs ou inadéquats de sa situation. Il y a donc un écart considérable entre la perspective globale sur laquelle doit théoriquement s'appuyer le plan de services individualisé et l'optique de la Loi sur la protection de la jeunesse.

La même discordance est perceptible sur le plan de la finalité de I'intervention. En contexte de protection, les préoccupations de développement personnel et d'intégration sociale cèdent le pas à l'élimination du danger, réel ou potentiel : «En protection de la jeunesse, l'intervention doit cesser dès que la sécurité et le développement (compris dans le sens de réponses aux besoins essentiels) de l'enfant ne sont plus compromis et dès que le risque de récurrence est minimalement jugulé » (Pinard, $1991: 30)$.

Le cadre restrictif de l'intervention de protection soulève la nécessité d'assurer un suivi auprès des enfants une fois que le mandat de protection est terminé. Comme le souligne le Groupe de travail pour les jeunes (1991), il est opportun que les autres ressources puissent s'impliquer lorsque le signalement n'a pas été retenu ou lorsque le dossier n'est plus pris en charge par la Protection de la jeunesse.

Cette éventualité soulève la question de la responsabilité de la coordination du plan de services. Si l'on conçoit l'application des mesures de protection comme l'étape initiale d'une stratégie d'intervention visant le développement personnel et l'intégration sociale de l'enfant, cette coordination devrait être faite par une personne qui pourra continuer d'accompagner l'enfant au terme de l'intervention de protection. Or, la pratique actuelle va plutôt dans le sens de confier la coordination au praticien chargé de l'application des mesures de protection. Bien que cette option reconnaisse le cadre légal et fasse ressortir l'importance d'articuler tous les services autour de l'objectif de protection, elle comporte également le risque de voir l'intervention s'arrêter une fois cet objectif minimal atteint, avec la possibilité de voir ces situations rebondir ultérieurement.

II n'y a pas que la nature de l'intervention de protection qui pose une difficulté aux principes du plan de services ; le cadre structurel de la pratique de protection, qui se caractérise par une segmentation des activités, en complexifie également l'application. 


\section{La segmentation des activités}

Le plan de services individualisé repose sur la nécessité d'assurer la concertation et la complémentarité des services, notamment en établissant une continuité entre les différentes étapes du processus d'intervention, à savoir : l'évaluation des ressources et des besoins, la détermination et la hiérarchisation des objectifs et la sélection des moyens d'action. Personne n'oserait évidemment nier l'importance de cette continuité, mais on conviendra qu'elle peut se révéler particulièrement difficile à réaliser dans un cadre de pratique marqué par la division et la spécialisation des tâches. Dans un contexte où des professionnels doivent travailler à la chaîne (Harvey, 1988b), si l'objectif de concertation et de complémentarité trouve toute sa pertinence, sa réalisation est d'autant plus problématique qu'un tel contexte constitue un terrain propice aux suspicions et aux rivalités (Pinard, 1991). Ainsi, au sein même de la Direction de la protection de la jeunesse, les conditions de pratique peuvent faire obstacle à l'établissement de la concertation entre les acteurs, et il est souvent nécessaire d'établir des mécanismes d'harmonisation entre les praticiens chargés de la réception et du traitement des signalements, ceux qui assurent l'évaluation et l'orientation et ceux qui sont chargés de l'application des mesures de protection. On ne s'étonnera donc pas que cette nécessaire complémentarité soit encore plus difficile à établir lorsqu'elle met en scène des intervenants qui viennent d'établissements avec des philosophies d'intervention variées et des mandats différents.

\section{Les particularités organisationnelles}

La diversité des philosophies et des mandats des organismes susceptibles d'être mis à contribution dans la protection d'un enfant constitue un obstacle non négligeable à l'élaboration et à la coordination du plan de services individualisé. Si, avec les années, les intervenants ont réussi à concilier la pratique sociojuridique avec les valeurs traditionnelles du travail social (Boucher et al., 1985 ; Mercier, 1991 ; Yelaja, 1971), dans la plupart des autres établissements l'adhésion volontaire du client à la démarche d'intervention constitue toujours une exigence, non seulement professionnelle mais également administrative, à la fourniture des services. Conséquemment, le recours à des mesures ordonnées risque de compromettre dès le départ la mise à contribution de collaborateurs extérieurs au CSS, limitant ainsi I'utilisation du plan de services individualisé aux situations débouchant sur des mesures volontaires. Comme le souligne avec à-propos le Groupe de travail sur l'application des mesures de protection de la jeunesse (1991a), l'instauration des plans de services individualisés ne sera pas facile, puisqu'elle « force les établissements et les intervenants à accepter de limiter leur autonomie, au nom de l'intérêt supérieur du client» (p. 156). Et les difficultés 
risquent d'être d'autant plus importantes que la notion même " $d^{\prime}$ intérêt supérieur du client » ne suggère pas nécessairement une voie unique d'action.

L'autonomie administrative des établissements combinée à la marge de manœuvre des intervenants qui y travaillent ne peut que conduire à l'échec toute stratégie de concertation qui s'appuierait sur la contrainte. Seule une entente entre les acteurs, non seulement sur les objectifs à poursuivre mais également sur les moyens à mettre en œuvre pour y arriver, peut assurer la concertation et la cohésion dans l'action.

\section{Les impératifs d'ordre légal et la participation}

La participation du client à l'ensemble des étapes du processus d'intervention constitue un des éléments à la base du PSI. En tant qu'élément central du plan de services le client doit être associé directement à toutes les démarches entourant l'élaboration et l'implantation de celui-ci (Andele, 1990). D'une part, cette participation traduit le respect du client et la reconnaissance de ses capacités (MSSSQ, 1990) ; d'autre part, elle fait en sorte qu'il se sente concerné par les services qui lui sont proposés et qu'il en saisisse la pertinence et le fil conducteur (CSSQ, 1989).

Dans la mesure où le PSI s'appuie sur une vision globale de la réalité du client, cette participation doit également mettre à contribution les membres de son environnement qui sont les plus proches, soit sa famille et son milieu social proche. Cette condition est particulièrement importante sur le plan de l'intervention en protection où " le client est l'enfant en besoin de protection » (Groupe de travail sur l'application des mesures de protection de la jeunesse, 1991a). Dans ce contexte de pratique :

Assurer la primauté de la personne du jeune et de son individualité implique le respect de sa personnalité, de sa façon de vivre, de ses différences et des liens qu'il entretient avec son milieu. C'est également [...] favoriser sa participation et celle de ses proches, ses parents au premier chef (MSSSQ, 1990 : 38).

Or, cette participation n'est pas facilitée par le contexte sociojuridique qui encadre l'intervention de protection. Dans ce contexte, l'intervenant établit avec le client et ses proches, notamment les parents, un rapport « régi par une série d'articles de lois et de règlements qui constituent en fait des limites et des balises dans l'engagement de l'un ou de l'autre de ces acteurs » (Laforest et Redjeb, 1991 : 95). Le principe de la négociation entre partenaires égaux engagés dans la planification, la réalisation et la coordination d'un plan de services (Despins, 1990) trouve alors difficilement application. II en découle une plus grande difficulté à obtenir l'adhésion des personnes concernées aux services qui sont offerts et à mettre à contribution le réseau naturel de l'enfant et de la famille. 


\section{Quelques avenues de solution}

Ces observations sur les difficultés d'application du plan de services individualisé en contexte d'autorité ne rendent pas l'opérationalisation de cette démarche impossible pour autant. Grâce à certaines initiatives, il semble possible que cet instrument pave la voie à une plus grande concertation entre les acteurs et à une meilleure harmonisation des services. Au départ, il est nécessaire de considérer la protection de la jeunesse comme une responsabilité collective qui fait appel à la contribution de l'ensemble des ressources d'une communauté. Cela ne veut pas dire pour autant que toutes ces ressources doivent « être au service » de la Direction de la protection de la jeunesse. La responsabilité collective passe par la reconnaissance et le respect des mandats, des rôles et des modes de fonctionnement de chacun. Ainsi la contribution des ressources de la communauté doit s'inscrire dans un rapport de collaboration égalitaire et non de sous-traitance par rapport à la Direction de la protection de la jeunesse.

Il est également impératif que la réalité des enfants en besoin de protection soit évaluée dans une perspective élargie qui tient compte non seulement des dangers auxquels ils sont exposés ou des carences dont ils sont victimes, mais également des composantes enrichissantes de leur réalité familiale et sociale. Même lorsque la sécurité ou le développement d'un enfant est compromis, tout n'est pas que négatif ; il existe des éléments positifs sur lesquels les intervenants peuvent baser leur action.

La spécification, dès le départ, des objectifs visés par l'intervention et la clarification de la contribution attendue de chaque partenaire sont des mesures qui peuvent minimiser les risques de frictions. Si les intervenants de la DPJ peuvent imposer certaines contraintes aux parents par le mandat d'autorité dont ils disposent, ils ne peuvent en faire autant avec les organismes collaborateurs. Dans l'élaboration du plan de services, il est donc essentiel que chaque partenaire ait la conviction que la contribution qui lui est demandée n'a pas pour objectif caché de soulager un autre intervenant de responsabilités qui, à son avis, devraient lui revenir. Il doit réaliser que cette contribution s'inscrit dans une action collective dont l'objectif est d'assurer la sécurité d'un enfant et de favoriser son développement.

II nous apparaît essentiel que tous les acteurs soient présents au moment de l'élaboration du plan de services, afin que tous saisissent que la réponse aux besoins de l'enfant est le premier motif qui guide les décisions. Même si au départ on peut anticiper leur désaccord sur les mesures proposées, parents et enfant doivent participer à la démarche dès l'étape de planification. Il faudrait même envisager, dans le cas de mesures ordonnées, que la présence à la rencontre d'élaboration du plan de services individualisé soit inscrite à l'ordonnance, afin que les parents et l'enfant soient, sinon en accord, du moins informés de l'évaluation qui est 
faite de leur situation et des considérations qui sont à la base des services qui leur sont imposés.

Enfin, il est essentiel que soit facilitée la coordination des plans de services. Sur ce plan, Schwartz et ses collaborateurs (1982) suggèrent différentes stratégies pour accroître l'autorité du coordonnateur, dont : 1) l'établissement, entre les ressources concernées, de politiques et de procédures formelles et spécifiques liées au mandat et à l'autorité du coordonnateur, 2) l'attribution d'un pouvoir discrétionnaire au coordonnateur en ce qui a trait à la distribution des subventions aux organismes de services, 3) la sélection de coordonnateurs qui jouissent d'une grande crédibilité auprès des administrateurs et des intervenants. Partant, le statut professionnel du coordonnateur et sa préparation à assumer ce rôle doivent faire l'objet d'une attention spéciale. Selon Lamb (1980), le coordonnateur devrait posséder des compétences dans les domaines suivants : 1 ) connaissance des procédures de collaboration entre les organismes et maîtrise des habiletés liées au rôle de courtier, 2) habileté politique par rapport aux appareils bureaucratiques, 3) aptitudes pour le travail communautaire afin de situer et de développer, au besoin, les ressources pertinentes, 4 ) habiletés en intervention individuelle sur le plan de la relation d'aide et de l'évaluation de besoins, 5) capacité de reconnaître, de valoriser et d'assister le client dans son cheminement même si les progrès réalisés sont limités.

Outre l'exercice de l'autorité et la préparation reçue, d'autres facteurs peuvent influencer l'action du coordonnateur. Un de ces facteurs est la charge de travail. Une charge trop lourde réduit la qualité et l'efficacité de la coordination en diminuant la fréquence des contacts entre le coordonnateur et les clients et en confinant les interventions à des réponses aux situations de crise (Cohn et De Graaf, 1982 ; Intagliata, 1982). Un autre facteur tient à la supervision offerte. La supervision est généralement considérée comme une source de reconnaissance et de soutien et, à cet égard, elle peut contribuer à appuyer le coordonnateur dans la réalisation de fonctions peu valorisées sur le plan professionnel. Une supervision appropriée est donc essentielle à la motivation : "Adequate supervisory support is deemed essential in sustaining the motivation of case managers and preventing burnout » (Rubin, 1987 : 219).

Le recours au plan de services individualisé représente un tournant majeur en matière d'intervention dans le domaine de la protection de la jeunesse. On peut penser que la transition entre une pratique où le praticien chargé de l'application des mesures de protection agit seul et une pratique mettant à contribution les établissements du réseau et les organismes communautaires dans une stratégie concertée sera grandement influencée par les conditions dont bénéficieront les intervenants. 


\section{CONCLUSION}

L'effet combiné de l'émergence de nouvelles ressources communautaires et de la restriction des mandats confiés aux organismes institutionnels a posé la nécessité d'une plus grande harmonisation et d'une meilleure coordination des services. L'utilisation du plan de services individualisé constitue une des avenues privilégiées pour réaliser cette nécessaire concertation. Le recours à ce moyen risque d'avoir un impact non négligeable sur la pratique du travail social parce que, d'une part, la fonction des travailleurs sociaux les amène généralement à avoir des contacts avec un large éventail de ressources, les plaçant ainsi dans une position stratégique sur le plan de la coordination des services, et que, d'autre part, ils sont considérés comme les intervenants les mieux outillés, sur le plan de la formation, pour effectuer cette coordination. Or, cette éventualité ne sourit pas à tous les intervenants. Si certains y voient le risque d'une déqualification de leur pratique, d'autres sont davantage préoccupés par les difficultés d'actualisation des principes et de la démarche du plan de services individualisé. Dans ce texte, nous avons tenté de faire ressortir certaines de ces difficultés à la lumière des particularités de l'intervention en contexte d'autorité. Selon ce qui se profile à l'horizon, les travailleurs sociaux ne pourront pas se soustraire à l'utilisation du plan de services individualisé dans leur pratique ; il leur faut donc envisager dès maintenant la stratégie qui leur permettra d'en réaliser une intégration positive.

\section{Références bibliographiques}

ANDELE, K. (1990). Le plan de services individualisé : un outil de gestion des services. Montréal : Conseil régional de la santé et des services sociaux du Montréal métropolitain.

ASSOCIATION DES CENTRES DE SERVICES SOCIAUX DU QUÉBEC (1988). Plan de services individualisé. Montréal : ACSSQ.

AUSTIN, C.D. (1983). "Case management in long term mental care: options and opportunities », Health and Social Work, vol. 8, n' 1 : 16-30.

AuSTIN, M.J. (1981). Supervisory Management for the Human Services. Englewood Cliffs, NJ : Prentice-Hall.

BESHAROV, D.J. (1986). "Unfounded allegations : a new child abuse problem », The Public Interest, $\mathrm{n}^{\circ} 83: 18-33$.

BOUCHER, L., GOSSELIN, J. et D. GuAY (1985). " Le concept de protection : se questionner pour transformer la pratique ", Intervention, $\mathrm{n}^{\circ} 72: 56-62$.

CABOT, R.C., CANNON, I.M. et M.A. CANNON (1987). "Case Management», Encyclopedia of Social Work, $18^{\mathrm{e}}$ éd. Silver Spring, MD : National Association of Social Workers, p. 212-222. 
CENTRE DE SERVICES SOCIAUX DE QUÉBEC (1989). Formulation de plans de services individualisés en matière de protection de la jeunesse. Québec : Centre de services sociaux de Québec.

COHN, A.H. et B. DE GRAAF (1982). "Assessing case management in the child abuse field », Journal of Social Service Research, vol. 5, $\mathrm{n}^{\text {os }}$ 1-2 : 29-43.

CONSEIL RÉGIONAL DE LA SANTÉ ET DES SERVICES SOCIAUX DU MONTRÉAL MÉTROPOLITAIN (1989). Cadre théorique de plans de service aux jeunes en difficulté et à leur famille. Montréal : CRSSSMM.

CÔTÉ, R. et al. (1989). Guide d'élaboration des plans de services et d'interventions. Québec: Université Laval, GREDD.

DESPINS, S. (1990). "Des services planifiés », dans D. Boisvert, Le plan de services individualisé : participation et animation. Montréal : Agence d'Arc, p. 59-80.

FRANKLIN, J.L. et al. (1987). "An Evaluation of Case Management », American Journal of Public Health, vol. 77, $\mathrm{n}^{\circ} 6$ : 674-678.

GAGNÉ, F. (1991). Les intervenantes sociales et la protection de la jeunesse : fonctionnaires ou polices de la famille?, Nouvelles CSN, nº $329: 10-12$.

GARBARINO, J. et S.H. STOCKING (1980). Protecting children from abuse and neglect. Washington : Jossey-Bass.

GROUPE DE TRAVAIL POUR LES JEUNES (1991). Un Québec fou de ses enfants. Rapport du Groupe de travail pour les jeunes. Québec : ministère de la Santé et des Services Sociaux.

GROUPE DE TRAVAIL SUR L'APPLICATION DES MESURES DE PROTECTION DE LA JEUNESSE (1991a). La protection sur mesure : un projet collectif. Québec : ministère de la Santé et des Services sociaux.

GROUPE DE TRAVAIL SUR L'APPLICATION DES MESURES DE PROTECTION DE LA JEUNESSE (1991b). La protection sur mesure : un projet collectif. Annexe 2. Québec: ministère de la Santé et des Services sociaux.

HARVEY, J. (1988a). Rapport sur l'analyse des activités de réception et de traitement des signalements, et d'évaluation et d'orientation en protection de la jeunesse. Montréal.

HARVEY, J. (1988b). Rapport sur l'analyse des activités de réception et de traitement des signalements, et d'évaluation et d'orientation en protection de la jeunesse. Annexe 6. Montréal.

INTAGLIATA, J. (1982). «Improving the quality of community care for the chronically mentally disabled : the role of case management ", Schizophrenia Bulletin, vol. 8, no 4 : 655-674.

INTAGLIATA, J. et F. BAKER (1983). «Factors affecting case management services for the chronically mentally ill », Administration in Mental Health, vol. 11, $\mathrm{n}^{\circ} 2$ : 75-91.

JOHNSON, P.J. et A. RUBIN (1983). « Case management in mental health : a social work domain? », Social Work, vol. 28, no $1:$ 49-55.

JONES, M., MAGURA, S. et A. SHYNE (1981). "Effective practice with families in protective and preventive services: What works? », Child Welfare, vol. 60, $\mathrm{n}^{\circ} 2$ : 67-80.

KEMP, B.J. (1981). "The case management model of human service delivery», Annual Review of Rehabilitation, p. 212-238.

LABRECQUE-MARCEAU, M.P. (1987). "La coordination des plans de services », Santé mentale au Québec, vol. 12, no 1 : 108-116. 
LAFOREST, M. et B. REDJEB (1991). « Le service social et les conditions de sa pratique : un rapport à examiner », Service social, vol. 40, n $2: 89-104$.

LAMB, H.R. (1980). "Therapist-case managers : more than brokers of services", Hospital and Community Psychiatry, vol. $31: 763$.

LAurendeAu, R. (1989). Plan de services, plan d'interventions : Guide méthodologique. Hull : UQAH, $28 \mathrm{p}$.

LISTER, L. (1984). "System linkage dealing with sexual issues in a case management approach », Journal of Social Work and Human Sexuality, vol. 2, no 1 :33-51.

MERCIER, L. (1991). "Contexte d'autorité et judiciarisation : régression ou redéfinition novatrice de la pratique sociale professionnelle? », Service social, vol. 40, $\mathrm{n}^{\circ} 2: 43-53$.

Ministère de la SANTÉ et deS SeRVICES SOCIAuX DU QuÉBEC (1990). Cadre de référence sur l'orientation et l'organisation des centres de réadaptation pour jeunes en difficultés d'adaptation. Québec : Direction des communications.

MOXLEY, D.P. (1989). The Practice of Case Management. Beverly Hills, CA : Sage.

NADEAU, B. (1989). "Le case management au carrefour de I'intervention clinique et communautaire », Santé mentale au Québec, vol. 14, n 2 : 51-89.

PINARD, P. (1991). «La Loi sur la protection de la jeunesse et les travailleurs sociaux: impacts sur leurs valeurs, sur leur pratique et sur leur formation », Service social, vol. 40, $\mathrm{n}^{\circ} 2: 26-42$.

PYKE, J., CLARK, S. et J. WALTERS (1991). "Case Management », L'infirmière canadienne, vol. 87, $\mathrm{n}^{\circ} 1: 22-25$.

ROBERTS-DEGENNARO, M. (1987). "Developing case management as a practice model », Social Casework, vol. 68, n $8: 466-470$.

ROTHERY, M. et G. CAMERON (1990). Child Maltreatment : Expanding our Concept of Helping. Hillsdale, NJ : Lawrence Erlbaum Ass., publ.

ROTHERY, M. (1990). "Child maltreatment and the front-line worker », dans M. Rothery et G. Cameron (eds.), Child Maltreatment : Expanding our Concept of Helping. Hillsdale, NJ : Lawrence Erlbaum Ass., publ., p. 1-9.

RUBIN, A. (1987). "Case Management », dans N.A.S.W. (eds.), Encyclopedia of Social Work, Silver Spring, MD : N.A.S.W., $18^{\mathrm{e}}$ édition, p. 212-222.

SCHILLING, R.F., SCHINKE, S.P. et R.S. WEATHERLY (1988). "Service trends in a conservative era : social workers rediscover the past ", Social Work, vol. 33, $\mathrm{n}^{\circ} 1: 5-9$.

SCHWARTZ, S.R., GOLDMAN, H.H. et S. CHURGIN (1982). "Case management for the chronic mentally ill : models and dimensions », Hospital and Community Psychiatry, vol. 33, $\mathrm{n}^{\circ} 12$ : 1006-1009.

SEGAL, S.P. et J. BAUMOHL (1981). "Social work practice in community mental health », Social Work, vol. 26.

WHITTAKER, J. (1986). "Formal and informal helping in child welfare services: implications for management and practice », Child Welfare, vol. 55, $\mathrm{n}^{\circ} 1: 17-25$.

YeLAJA, S.A. (ed.) (1971). Authority and Social Work : Concept and Use. Toronto : University of Toronto Press. 